\title{
Percepciones, opiniones, hábitos, prácticas y preferencias de docentes y estudiantes ante la incorporación de las TIC en la secundaria del Colegio Calasanz
}

Gloriana Anchetta Meza

Productora académica, Programa de Producción de Materiales Didácticos Escritos, UNED; ganchetta@uned.ac.cr

Recibido: 20 de abril del 2016

Corregido: 01 de julio del 2016

Aceptado: 06 de julio del 2016

\begin{abstract}
Resumen
El propósito del artículo es presentar las opiniones, hábitos, preferencias y prácticas con respecto a las tecnologías dentro del proceso de enseñanza-aprendizaje de los estudiantes y docentes de secundaria del Colegio Calasanz con el fin de detectar aspectos positivos y por mejorar en relación con las tecnologías de la información y la comunicación (TIC).

Los datos fueron recolectados, analizados y contrastados a través de una metodología interpretativa para conocer las interacciones entre estudiantes, profesores y elementos del entorno relacionados con las tecnologías. Se observó que los estudiantes usan frecuentemente las tecnologías en su cotidianeidad y, al mismo tiempo, exigen nuevas metodologías didácticas que les permiten ser más flexibles, dinámicos y aprovechar mejor las TIC. Por su parte, los docentes, pese a considerar que el uso de las TIC en el contexto educativo es importante, no cuentan con las condiciones de tiempo y de capacitación adecuadas para hacerlo con calidad. Por ello, se recomienda que la institución implemente una metodología pertinente y una propuesta de capacitación innovadora con el fin de mejorar el proceso de enseñanza-aprendizaje y adaptarse a las necesidades y características de los estudiantes.
\end{abstract}

Palabras clave: educación secundaria, herramientas tecnológicas, opiniones de docentes y estudiantes, calidad educativa, hábitos tecnológicos.

\section{Abstract \\ Perceptions, opinions, habits, practices and preferences of teachers and students about the incorporation of ICT in the Calasanz secondary school}

The purpose of this article is to present the opinions, habits, preferences and practices regarding technologies in the teaching-learning process of the students and teachers of secondary Calasanz School, in order to detect positive aspects and improve in relation to information and communications technology (ICT).

The data were collected, analyzed and verified through an interpretative methodology to understand the interactions between students, teachers and environmental elements related to technologies. It was observed that students frequently use technologies in their daily lives and at the same time, demand new teaching methodologies that allow them to be more flexible, dynamic and make better use of ICT. Meanwhile, teachers, despite considering that the use of ICT in the educational context is important, do not have the time conditions and adequate training to achieve it with quality. Therefore, 
it is recommended that the institution implements an appropriate methodology and a proposal to innovative training in order to improve the teaching-learning process and adapt to the needs and characteristics of students.

Key words: high school education, technological tools, opinions of teachers and students, educational quality, technological habits.

\section{INTRODUCCIÓN}

La sociedad del conocimiento en la que estamos insertos ha variado la forma en que se busca información, se transmite y se comunica. Internet aloja innumerables contenidos multimediales y con múltiples posibilidades de interactuar y compartir: así, los individuos encuentran más atractivo y útil aprender mediante estos medios que por los tradicionales (Maglione y Varlotta, 2010).

El sistema educativo debe adaptarse al nuevo contexto, por lo que es preciso que los estudiantes desarrollen habilidades para saber cómo seleccionar la información adecuada, cómo aplicarla y cómo convertirse en productores y no en reproductores de conocimientos. Es indispensable, además, incorporar las tecnologías en el proceso educativo, pero mediante un diseño didáctico integrado. Además, es fundamental conocer quiénes son los estudiantes, sus características, necesidades y gustos al respecto, así como las posibilidades y conocimientos de los docentes. De lo contrario, es probable que su ejecución sea incorrecta y no aporte cambios significativos en el proceso educativo.

En este sentido, si una institución educativa pretende insertar las tecnologías, deberá realizar una investigación acerca de las características (opiniones, hábitos, preferencias y prácticas) de estudiantes y docentes, de manera que se aclare con qué se cuenta y cuáles acciones deben tomarse antes de la incorporación.

En este artículo se exponen los resultados de la investigación que se llevó a cabo en el Colegio Calasanz. La institución sintió la necesidad de actualizarse e innovar en estrategias didácticas por medio de las tecnologías y, como primer paso, apostó por realizar el estudio. Así, se detectaron los aspectos positivos y por mejorar antes de planificar la nueva propuesta educativa.

\section{ESTADO DE LA CUESTIÓN}

El Fondo de las Naciones Unidas para la Infancia (UNICEF) en el 2010 hace hincapié en la importancia de que los jóvenes adquieran capacidades y herramientas llamadas "competencias de empleabilidad", que se refieren a capacidades para desenvolverse en el campo universitario y laboral futuro. Entre estas se encuentran las habilidades de comunicación, la solución de problemas, la capacidad de abstracción, el uso de las tecnologías, el trabajo en grupo y la responsabilidad por los resultados.

Por su parte, Area (2010), en un estudio realizado sobre el proceso de integración pedagógica de las TIC en secundaria, determinó que los alumnos están muy motivados con las actividades que se desarrollan en el aula virtual. Ellos han entrado en contacto con un medio de aprendizaje que muestra la peculiaridad de combinar la imagen, el texto y el sonido, lo cual ofrece nuevas posibilidades de aprendizaje. Sin embargo, el profesorado ha expresado su preocupación debido a que el alumnado puede perder esta gran motivación hacia el trabajo con las TIC si existen medios digitales inadecuados en relación con las características de los estudiantes. 
Según un estudio realizado en Barcelona en el 2007 (Raventós, 2007), en una secundaria se incorporaron búsquedas en bases de datos en internet y en el uso de diferentes programas se observó una mejora en el rendimiento de los alumnos responsables y motivados. Para Ayllón (2010) "el alumnado tiene la opción de poder elegir sus propios itinerarios de descubrimiento del conocimiento con un simple clic del ratón, abriendo y cerrando ventanas de información textual o audiovisual" (p. 2). Se ha estudiado que el uso de las TIC permite abrir la institución al entorno social para educar de acuerdo con las necesidades contextuales, permitiendo el cuestionamiento y la discusión de los estudiantes ante las situaciones actuales de su región. Empero, se ha observado que todavía existe resistencia por parte de los centros educativos hacia el acceso a las tecnologías.

Ahora bien, la UNESCO (2013) señala que la zona centroamericana no cuenta con suficientes docentes capacitados para poder integrar las TIC de forma eficiente en los procesos educativos y que, en ocasiones, los programas de formación se orientan a que los profesores aprendan cómo adaptar contenidos y no al desarrollo de habilidades informáticas necesarias para la sociedad del conocimiento. La UNESO (2013) destaca a Costa Rica con ciertos aspectos como la inclusión de programas educativos mediante tecnologías, aunque también se subraya la falta de capacitación a los docentes en el tema y la escasa educación mediada por computadora en el nivel de secundaria.

Por otra parte, se vuelve pertinente analizar algunos estudios que han detallado las actitudes, opiniones y prácticas respecto a la tecnología empleada por los estudiantes costarricenses. Según Fournier y Grillo (2010), muchas veces los adolescentes no "miden" el tiempo empleado en internet. Además, de acuerdo con Pérez, Blanca, Brenes, Quesada, Rumoroso y Torres (2008) los adolescentes tienden a recurrir al uso de las TIC, mostrando competencia y facilidad en su uso. En este sentido, sobresale el uso del celular y de internet fijo y son más importantes aquellas actividades o prácticas que se relacionan con socialización, comunicación, mensajería y entretenimiento, tales como redes sociales, mensajería instantánea y descarga de información.

Entre las razones que motivan a los adolescentes a utilizar internet se encuentran la existencia de recursos y servicios de vanguardia, la capacidad de construir un perfil con el que quieren ser "vistos" por los demás e interactuar con individuos que a veces trascienden los vínculos primarios físicos (Fournier y Grillo, 2010). Por su parte, respecto de los sitios web, se encontró que en la mayoría de los estudiantes existe una tendencia a visitar sitios que de alguna forma garantizan rapidez y disponibilidad de información y servicios (Pérez et al., 2008).

Se encontró que el uso de internet con fines académicos o educativos es la actividad menos importante para los jóvenes en comparación con aquellas que se relacionan con socializar y comunicar. El hallazgo se puede relacionar con la falta de materiales y plataformas interactivas destinadas para adolescentes y niños (Fournier y Grillo, 2010). De hecho, de acuerdo con el informe del PROSIC (2009), muchos de los jóvenes opinan que el colegio no apoya significativamente el uso de tecnologías, lo que supone que en el hogar el acceso es más libre e intenso. No obstante, la mayoría considera que las tecnologías son una herramienta básica para el estudio y para su futuro laboral y académico, pero que casi no las usan, emplean más los libros.

Según el Informe del Estado de la Nación del 2015, los docentes presentan un alto acceso a las TIC; pero hay una brecha importante entre el uso y la aplicación dentro del salón de clases. Se determina que más de la mitad no conoce sobre la metodología y la didáctica en este campo, además de las deficiencias en cuanto al equipo tecnológico. 


\section{MARCO METODOLÓGICO}

Como se explicó en el punto anterior, la investigación muestra las opiniones y las prácticas de los estudiantes y profesores del Colegio Calasanz. El proceso se logró mediante la recopilación de datos relacionados con la investigación y que, a su vez, se utilizaron para respaldar los resultados obtenidos.

Respecto a los estudiantes, se consideró una muestra de 161 alumnos de secundaria, con una sección por año (7A, 8B, 9C, 10A y 11B). Cada sección fue elegida al azar, de acuerdo con la disponibilidad para realizar el cuestionario en el momento en que se iba a aplicar. En cuanto a los profesores se trabajó con toda la población de docentes de las distintas asignaturas impartidas.

De acuerdo con el análisis bibliográfico y con entrevistas a especialistas (Anchetta, 2015), se seleccionaron las variables por incluir en los cuestionarios. Además, se procedió a definirlas como se observa en el cuadro 1:

CUADRO 1

Variables en estudio y su definición conceptual y operacional

\section{Variable}

Hábitos de uso de las tecnologías

\section{Calidad del} aprendizaje aprendizaje

Contenidos didácticos
Definición conceptual

Comportamientos repetidos frecuentemente al aplicar las diferentes funciones que se derivan del uso de la computadora e internet, asociadas con labores académicas y extra académicas de los estudiantes y profesores (Henríquez y Organista, 2009).

Educación que permita que los alumnos aprendan a aprender, de manera pertinente y eficiente, al mismo tiempo que busca que sean seres sociales motivados, autónomos y con capacidad de impactar en su comunidad (Bravslasky, 2004).

Conjunto de conocimientos científicos, habilidades, destrezas y teoría que deben servir como base para la construcción del aprendizaje de los estudiantes (Organización para la cooperación y desarrollo económicos, 2010).

Conjunto de acciones diseñadas, planificadas y ejecutadas de manera que los estudiantes logren los objetivos didácticos planteados, a la vez que permiten la transformación de los contenidos en construcción de aprendizajes (Penzo, Fernández, García, Gros, Pagés, Roca, Vallés y Vendrell, 2010).

Evaluación de los aprendizajes

Proceso en el que se identifica, obtiene y proporciona información útil y descriptiva sobre el impacto de un programa o actividad determinada en el estudiante. Su fin es servir de guía para la toma de decisiones, solución de problemas y la promoción de la comprensión del proceso de enseñanzaaprendizaje (Joint y Stufflebeam, citados por Clavijo, 2008).

Uso de las Articulación de las herramientas digitales de la información y comunicatecnologías en el aula ción en el proceso y las prácticas de enseñanza y aprendizaje (Sánchez, s.f.).

Formación docente en innovación y TIC
Proceso de aprendizaje continuo por parte de los docentes, aprenden a aprender y transforman sus conocimientos con el objetivo de actualizarse en nuevas tendencias pedagógicas y en el uso de las tecnologías como herramientas didácticas, para así optimizar su labor con los estudiantes (Ávalos, 2011).
Definición operacional

Análisis de las prácticas de uso de las tecnologías en los estudiantes y profesores de secundaria del Colegio Calasanz.

Análisis de la opinión de los estudiantes y profesores de secundaria del Colegio Calasanz sobre los elementos relacionados con la calidad de aprendizaje.

Análisis de las opiniones de los estudiantes y profesores de secundaria del Colegio Calasanz sobre las características de los contenidos didácticos utilizados en clase.

Análisis de la opinión de los estudiantes y profesores de secundaria del Colegio Calasanz sobre la implementación de actividades de aprendizaje.

Análisis de la opinión de los estudiantes y profesores de secundaria del Colegio Calasanz sobre la evaluación de los aprendizajes.

Análisis de la opinión de estudiantes y profesores de secundaria del Colegio Calasanz sobre el uso y la integración de las TIC en el proceso de enseñanza-aprendizaje.

Análisis de las características de la formación y la actualización docente de los profesores de secundaria del Colegio Calasanz.

Fuente: Anchetta (2015). 
A partir de la definición de las variables, se elaboraron los cuestionarios para recolectar los datos mediante preguntas abiertas y cerradas. Los cuestionarios para estudiantes y docentes contaron con instrucciones generales, los datos de perfil sociodemográfico y las secciones de preguntas que responden a las diferentes variables por medio de ítems cerrados y abiertos que permitieron conocer sobre opiniones, gustos, preferencias, prácticas, actitudes y comportamiento con respecto al proceso de enseñanzaaprendizaje y el uso de las tecnologías en las aulas de la institución educativa.

El análisis se realizó gracias a la técnica de triangulación de los datos, los cuales se analizaron y se cuantificaron de acuerdo con la clasificación de la respuesta brindada. Luego se tabularon en el programa Microsoft Excel y se realizó una distribución porcentual según la respuesta a cada una de las preguntas planteadas.

En cuanto a las preguntas abiertas, se realizó un análisis por categorías con el fin de lograr realizar comparaciones, contrastes e interpretaciones.

\section{RESULTADOS Y DISCUSIÓN}

Para facilitar el análisis de los resultados, se separaron según las variables planteadas anteriormente. A continuación se presentan los resultados por variable.

\section{Hábitos de uso de las tecnologías}

De acuerdo con el cuestionario aplicado a los estudiantes, prácticamente todos cuentan con acceso a internet tanto en su hogar como en el celular, además, invierten una cantidad importante de horas al día accediendo a la web y aprovechando diferentes actividades. Según los resultados (figura 1), en orden descendente los estudiantes utilizan las redes sociales, la búsqueda de información como pasatiempo, la mensajería instantánea, los videojuegos, el correo electrónico y la búsqueda de información para tareas o trabajos.

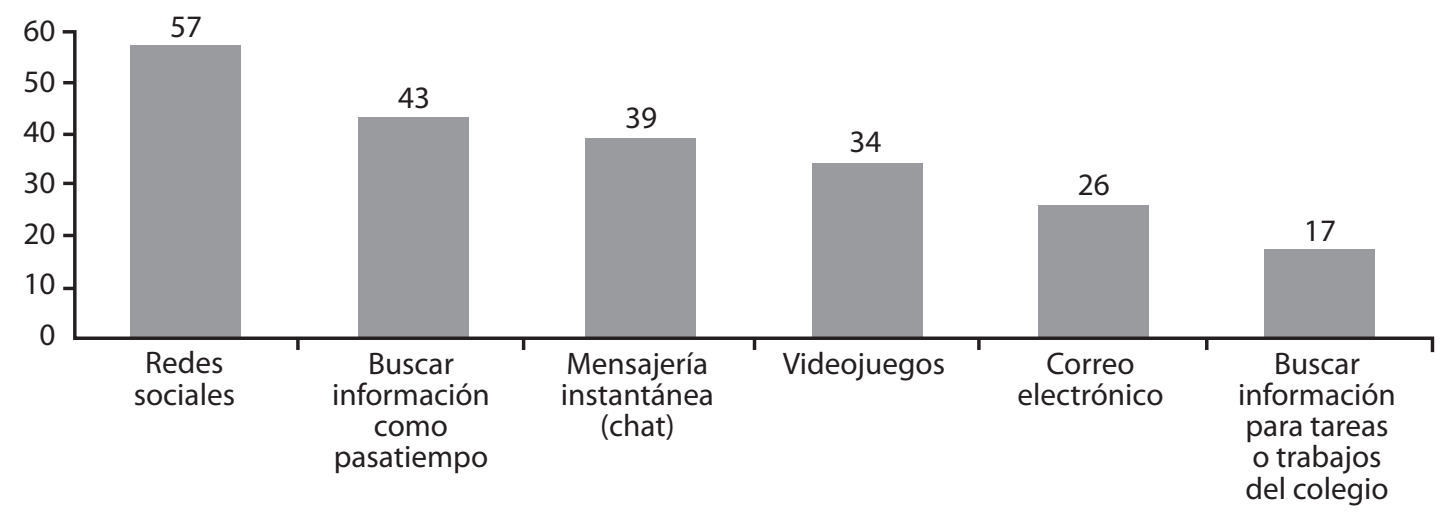

Figura 1. Cantidad de estudiantes de secundaria del Colegio Calasanz que utiliza las diferentes tecnologías. Fuente: Anchetta (2015). 
Se trata así de una realidad muy importante de tomar en cuenta, tanto por la institución educativa como por los docentes, ya que los estudiantes ejercen ciertas prácticas distintas a las tradicionales, las cuales involucran las tecnologías como, por ejemplo, las formas en que llevan a cabo el diálogo, la comunicación y la construcción de relaciones (Mella, 2003), lo cual repercute directamente en la construcción de su ser social. Por ejemplo, los estudiantes muestran un uso alto de redes sociales y mensajería instantánea, que modifica la manera en que se comunican y se relacionan entre ellos mismos y con el entorno.

Aunque en menor grado, en comparación con el uso de la mensajería instantánea y redes sociales, los estudiantes manifestaron prácticas para la búsqueda de información por medio de las tecnologías, tanto para trabajos o tareas del colegio como para entretenimiento y ocio, siendo esta última mayor que la anterior. Esto se relaciona con el hecho de que, según expertos como Ayllón (2010), los adolescentes están actualmente expuestos a un ambiente cambiante que permite el acceso a la información con muy poco esfuerzo y en tiempo real; además, el mismo especialista expresa que los jóvenes acceden a información de forma audiovisual y textual, lo cual es más apetecido por esa población.

Adicionalmente, las TIC están presentes en las diferentes aristas de la actividad humana y, por consiguiente, se han de convertir en parte fundamental del proceso de enseñanza-aprendizaje, permitiendo trascender el tiempo horario de las clases presenciales y las cuatro paredes del aula.

\section{Calidad del aprendizaje}

De acuerdo con la definición de esta variable (cuadro 1), el componente de motivación es fundamental en esa concepción de calidad del aprendizaje. Conforme con los resultados encontrados en los cuestionarios para los estudiantes, la mayoría (64\%) se siente motivado por aprender y en ocasiones se aburren en clases; también hubo un número importante de alumnos que manifestaron que casi siempre o siempre poseen ese sentimiento (12\% y 17\%). Es un resultado "regular" para la institución; empero, en un entorno educativo que pretende ser de calidad, se debe promover que los estudiantes estén lo más motivados posibles.

¿Cómo lograrlo? Los docentes deben planificar sus lecciones tomando en cuenta que sean motivadoras para sus estudiantes (Boekaerts, 2006). Sin embargo, en los cuestionarios para docentes, la mitad de ellos contestaron que regularmente no planifican sus lecciones para que sean motivadoras.

Una de las maneras que sería útil para aumentar la estimulación y atención de los estudiantes es la incorporación de las TIC en el aula. De acuerdo con el estudio realizado por la Fundación PANIAMOR (Fournier y Grillo, 2010), los estudiantes se sienten motivados para utilizar las TIC por los servicios y recursos de vanguardia; además, por las posibilidades de comunicación e interacción. Alrededor de $50 \%$ de la muestra expresó en los cuestionarios que para mejorar las lecciones el uso de herramientas tecnológicas es un apoyo importante; por ejemplo, mediante el uso de videos, estimuladores visuales, audios, videojuegos, páginas web, entre muchas otras opciones.

Otro punto importante que mencionaron los estudiantes fue con respecto a la actitud de los docentes. Algunos alumnos mencionaron que un modo de mejorar su aprendizaje sería que los profesores los motivaran: les dieran consejos para superarse en la vida y en los estudios, además de enseñarles con paciencia y utilizando técnicas didácticas diferentes. Cabe destacar que $90 \%$ de los estudiantes señalaron este último factor como fundamental para apoyar su aprendizaje; por ejemplo, al realizar juegos, debates, dinámicas, competencias, entre otras actividades.

La aplicación de aprendizajes a contextos y a problemáticas reales facilita significativamente los aprendizajes de acuerdo con la teoría del aprendizaje situado y con la teoría de la instrucción anclada (Rioseco 
y Romero, s.f.). En ese sentido, la mayoría de los estudiantes manifestaron que solamente a veces los aprendizajes son útiles y aplicables a su vida diaria.

Al respecto, alrededor de $80 \%$ de los profesores presentan los contenidos considerando que sean relevantes y aplicables a la vida diaria de los estudiantes. No obstante, se requiere que sea una práctica permanente en el proceso de enseñanza-aprendizaje, de manera que la experiencia sea de mayor calidad.

Una forma de lograr que los aprendizajes sean relevantes y aplicables a la vida diaria es mediante las TIC, por cuanto son herramientas que permiten el acceso a información actualizada a cada segundo; asimismo, son recursos que facilitan la creación de conocimientos y de investigación, pero que deben estar orientados a resolver problemáticas del entorno propio de la actividad educativa. Un alto porcentaje de los estudiantes expresó la importancia de la aplicación de lo aprendido a contextos y situaciones reales e ir más allá de la memorización y repetición de conceptos abstractos que no son útiles para ellos.

Así también, la calidad del aprendizaje implica que los estudiantes "aprendan a aprender", como lo indican expertos en el Informe de la comisión internacional para el siglo XXI de la UNESCO ya desde el año 1996. No basta, en ese sentido, que los conocimientos se memoricen y se repitan en una prueba memorística, sino que deben servir para formar a un estudiante íntegro, capaz de desenvolverse e incidir positivamente en su sociedad, así como en su futuro profesional y personal (Tedesco, 2011).

De acuerdo con el análisis de los resultados, la mayoría de los estudiantes entrevistados aseguró que a veces olvidan los aprendizajes luego de ser evaluados; además, alrededor de 30\% mencionó que casi siempre o siempre los olvidan. Así también, la mayoría de los estudiantes respondió que solamente a veces sienten deseos de seguir aprendiendo fuera del tiempo lectivo y un porcentaje destacable mencionó que casi nunca o nunca siente estos deseos. Entonces, ¿se están llevando acciones para que los aprendizajes sean permanentes?, ¿se está colaborando a construir habilidades necesarias para la sociedad del conocimiento como la crítica, la investigación, la flexibilidad y el "aprender a aprender"?

Según las investigaciones hechas por Prensky (2010), los estudiantes están cambiando: sus características y la forma en que aprenden es diferente al modelo tradicional, lo cual implica que la metodología de enseñanza debe variar con el fin de motivar al estudiante, interesarlo y hacerlo partícipe activo de su propio proceso de aprendizaje, al mismo tiempo que desarrolla habilidades relevantes para su futuro profesional.

¿Cómo conseguirlo? Las TIC son herramientas que, al ser adecuadamente utilizadas, poseen el potencial de cambiar la manera en que se lleva a cabo el proceso de enseñanza-aprendizaje. De acuerdo con Prensky (2010), los nativos digitales son aquellos que nacieron luego de 1980 y que han crecido rodeados de los nuevos avances tecnológicos, producto de la exposición e inmersión al uso de computadoras, videos, videojuegos, música digital, telefonía móvil y otro tipo de entretenimientos afines.

Así, los estudiantes de secundaria del Colegio Calasanz, cuya edad oscila entre los 12 y los 18 años, son nativos digitales. Por lo tanto, hay que atraerlos y proponerles alternativas diversas, tal como los mismos alumnos lo indican. El plantear estrategias didácticas innovadoras y tecnológicas donde se les otorgue más independencia, motivación y conocimientos relevantes e interesantes a los estudiantes servirá para la construcción de aprendizajes más permanentes y de mejor calidad (Bono, 2011).

\section{Contenidos}

Ante el cambio en la concepción del proceso educativo (Pegalajar, 2015), el aprendizaje es actualmente un fenómeno que debe llevarse a cabo de forma activa. Una de las condiciones para lograrlo es generar en los estudiantes interés e involucramiento en el proceso de aprendizaje para que trabajen y 
se desempeñen como verdaderos protagonistas. ¿Cómo conseguirlo? Según la UNESCO (2004) por medio de la presentación de contenidos que involucren diferentes características como los diversos estilos de aprendizaje, el contexto social y la realidad de los estudiantes, además de ser interesantes, significativos y relevantes.

Lo anterior es una premisa indispensable para lograr las metas pedagógicas propuestas en el Colegio Calasanz, donde pretenden el desarrollo de habilidades, destrezas y aprendizajes basados en los contenidos presentados y analizados durante las lecciones. En consonancia con la investigación realizada, alrededor de $80 \%$ de los docentes presentan contenidos mediante diversos recursos como videos, audios, esquemas, imágenes y texto, entre otros. Así también, todos los profesores afirmaron que trabajan con contenidos actualizados y relevantes, aunque $45 \%$ de ellos expresó que no lo hace siempre.

En cuanto al abordaje de contenidos que tomen en cuenta el contexto en el que se desarrolla el proceso de enseñanza-aprendizaje específico del Colegio Calasanz, todos los docentes manifestaron trabajar de este modo; sin embargo, alrededor de $80 \%$ de las respuestas expresadas por los docentes arrojaron que no lo hacen siempre.

Al analizar los resultados, se observó que los docentes se interesan en trabajar contenidos actualizados, relevantes y acordes con contexto de los estudiantes de la institución. Esto constituye una ventaja pues es evidente el compromiso con el desarrollo de actualización y con el estudio del entorno; pero de acuerdo con los resultados analizados, tal práctica no se desarrolla siempre. Entonces, ¿se estarán ofreciendo contenidos adecuados para el aprendizaje de los nativos digitales?, ¿se están aprovechando las características de los estudiantes de manera que se facilite la comprensión de los contenidos y el aprendizaje de calidad?

Por otra parte, ¿por qué no se está trabajando con contenidos actualizados y contextualizados en todas las ocasiones?, ¿será que el docente no cuenta con el tiempo o la libertad suficientes durante la lección para conseguir e incorporar datos actualizados y del entorno? Los mismos docentes expresaron que la falta de tiempo es un obstáculo para la innovación en las estrategias didácticas, tal como se observó en otras investigaciones como la elaborada por Alemán, Sancho y Gómez (2015).

La mayoría de los estudiantes (91\%) manifestaron que solamente a veces los contenidos están actualizados, se presentan mediante diversos recursos tecnológicos y se relacionan con su vida diaria y con la realidad de su país.

Por otro lado, de acuerdo con las teorías de Prensky sobre los nativos digitales, es necesario que los contenidos didácticos "tradicionales" se expongan utilizando diversos software y hardware, que incluyan diferentes recursos como audios, imágenes, videos, etcétera; los estudiantes nativos digitales aprecian mejor la información presentada gracias a dichas herramientas, pues su estructura cerebral ha sufrido cambios al respecto (Prensky, 2010).

\section{Actividades para los estudiantes}

Ahora bien, como se observó en la sección anterior, los estudiantes buscan que los contenidos posean ciertas características específicas que impacten positivamente en su aprendizaje. Sin embargo, el proceso educativo debe ir más allá de los contenidos y el docente debe proponer actividades que permitan que el estudiante construya el conocimiento, tal como lo indica el Portal Educativo de las Américas (2011) en la siguiente cita: 
No es posible aprender y apropiarse de los contenidos solamente acudiendo a la lectura de los materiales didácticos. No es posible solamente "transmitir" los conocimientos, sino que estos deben ser construidos y apropiados por los alumnos. La información que no es apropiada ni construida, permanece inerte y no disponible para aplicar en otras situaciones o contextos. (Portal Educativo de las Américas (2011), citado por Meza (2013, p. 30)

El párrafo anterior constituye una premisa indispensable para lograr los objetivos de cada materia del Colegio Calasanz. Por eso, se debe tener mucho cuidado a la hora de proponer actividades: debe tomarse en cuenta siempre el propósito general del curso y el propósito de formación integral que se persigue. Asimismo, es importante que se planteen tanto actividades individuales como colaborativas para enriquecer y complementar el aprendizaje (Meza, 2013).

De acuerdo con la investigación realizada, en cuanto al tema de las actividades que se proponen a los estudiantes, los profesores las planifican en $80 \%$ de los casos y tratan de lograr un aprendizaje significativo que fomente la solución de problemas y que sea motivador, el restante $20 \%$ de los docentes planifica únicamente a veces las lecciones y no toman en cuenta que se logre un aprendizaje significativo.

En una menor proporción (alrededor de 60\%) se incentivan las actividades colaborativas, el trabajo basado en proyectos y la utilización de casos reales como base de la actividad. También en una proporción menor se fomenta el diálogo, la discusión, la reflexión y la criticidad. En cuanto a la planificación de actividades en las que se incentive la diversión, o se tomen en cuenta las características individuales, el porcentaje fue inferior a 40\%. Lo expuesto se puede observar en la figura 2.

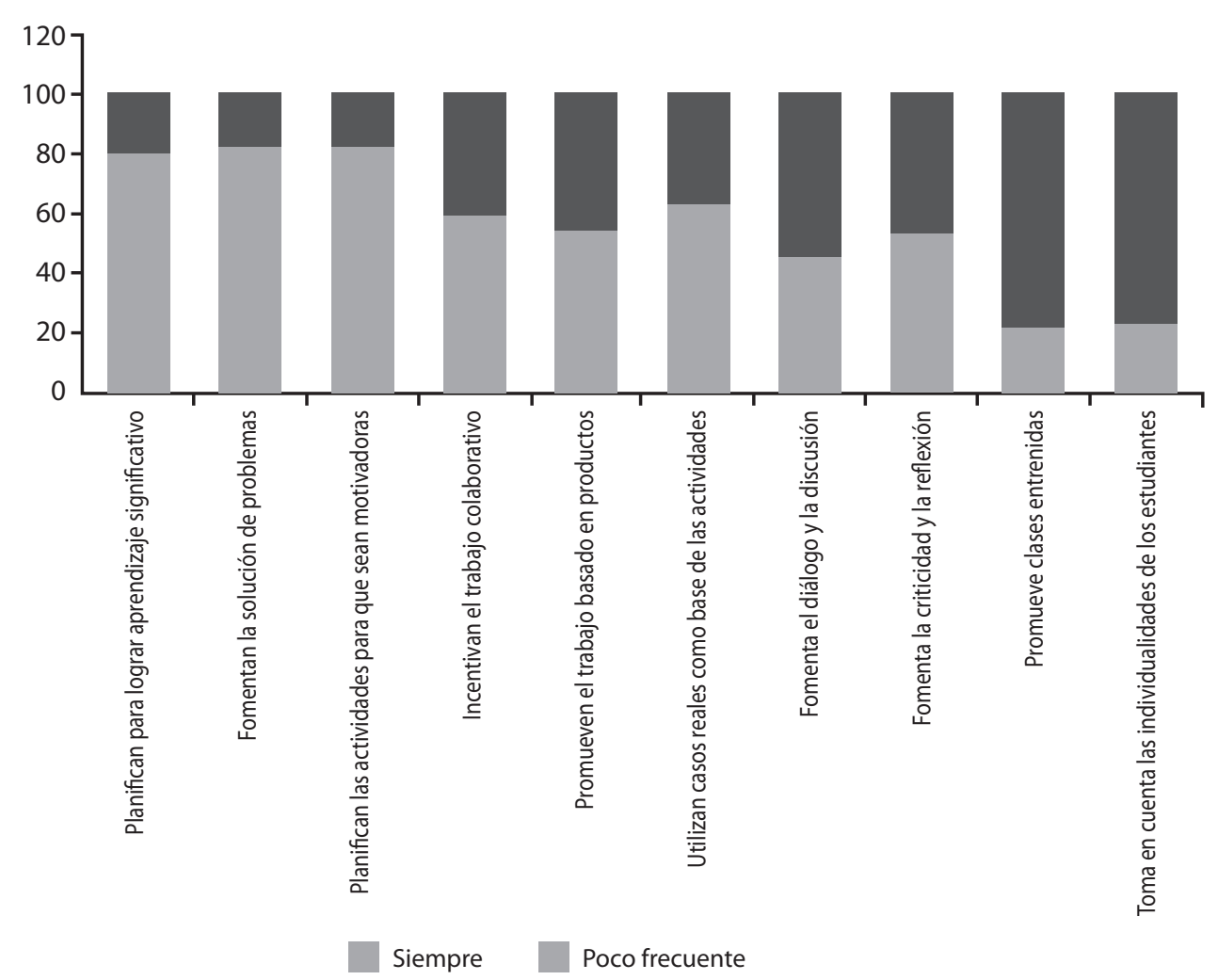

Figura 2. Distribución de los profesores de acuerdo con las características de las actividades ejecutadas. Fuente: Anchetta (2015). 
Se observa que varios de los profesores se interesan por planificar las actividades, que sean motivadoras y se logren aprendizajes significativos. Es una ventaja pues se observa el compromiso que los docentes del colegio sienten con sus alumnos. Empero, son menos los profesores que fomentan el trabajo en grupo, proponen casos reales en las actividades, incentivan el entretenimiento mientras aprenden o fomentan la discusión y la reflexión.

Entonces, ¿realmente lograrán aprendizajes significativos si casi no se da la oportunidad de discutir o de proponer?, ¿serán motivadoras las clases si los estudiantes indican que solo a veces se toman en cuenta sus opiniones y cuestionamientos? Además, ¿por qué será que no se fomenta mucho el trabajo colaborativo, las discusiones ni las actividades lúdicas, entre otras?, ¿el profesor no cuenta con el tiempo suficiente para hacerlo dentro de su lección de dos horas?, ¿será que no conoce herramientas que le ayuden a plantear este tipo de actividades? Los mismos profesores expresan en las respuestas a los cuestionarios que el tiempo y la obligación de cumplir con las exigencias del MEP son obstáculos para realizar actividades con TIC y que, además, no cuentan con el conocimiento suficiente para hacerlo.

Desde el punto de vista de los estudiantes, la mitad de ellos expresan que solo algunas veces se les brinda la oportunidad de reflexionar, criticar, discutir, proponer ideas, investigar o trabajar en grupos, lo cual implica que podría no lograrse el aprendizaje significativo que los profesores se proponen. Un alto porcentaje (78\%) indicó que solo pocas veces se presentan actividades lúdicas. Por un lado, los mismos estudiantes expresan que los profesores podrían mejorar sus lecciones si sus clases fueran más activas, menos aburridas, con puesta en común, con trabajos en grupo, con actividades de opinión, de participación, crítica, discusión y debate, además de considerar herramientas tecnológicas y clases innovadoras.

En síntesis, es importante que el estudiante sea el centro del aprendizaje, por ello los docentes deben diseñar propuestas didácticas que inviten a tomar posesión de su propio aprendizaje, desarrollar el pensamiento crítico y creativo, procesar información, tomar decisiones, manejar conflictos y focalizar el trabajo en colaboración.

De lo expuesto en este apartado acerca de la propuesta de actividades en el colegio, se destaca la necesidad de capacitar a los profesores en estos temas, de modo que comprendan que las actividades deben ser más propositivas y más interesantes para los alumnos. En ese sentido deben comprender que la utilización de las TIC representa una ayuda para facilitar la propuesta de ese tipo de actividades y no un esfuerzo adicional a lo que solicita el MEP. Asimismo, la utilización de las TIC no es una pérdida de tiempo en una lección de dos horas, sino que deben integrarse a todo el proceso y específicamente para la realización de actividades con las que se logrará un aprendizaje significativo y más ágil, y propuestas de enseñanza-aprendizajes de calidad.

\section{Evaluación de los aprendizajes}

Al analizar los datos acerca de la evaluación de los aprendizajes, la mayoría de los profesores solicitan a los estudiantes la memorización y la repetición de los contenidos en los exámenes. Además, indicaron que alrededor de $50 \%$ utiliza casos reales a la hora de plantear los exámenes. También los estudiantes reafirmaron lo indicado por los profesores pues casi siempre o siempre deben memorizar los contenidos y repetirlos en las pruebas de evaluación. Los resultados anteriores permiten concluir que la aplicación de pruebas evaluativas en que la memoria y la repetición de contenidos son componentes principales es común en el proceso de enseñanza-aprendizaje de la secundaria en estudio (H. Sánchez, comunicación personal, 19 de julio de 2014). Sin embargo, 40\% de los estudiantes expuso que algunas veces los docentes les solicitan la realización de proyectos en lugar de solamente exámenes. 
Otro elemento importante que se indagó fue la posibilidad de mejorar la nota mediante la realización de algún trabajo o asignación extra. Solo $18 \%$ de los profesores indicó que a veces daban la posibilidad de mejora, los estudiantes reafirmaron lo aseverado.

Del análisis efectuado se desprenden las siguientes aseveraciones:

La calificación de los estudiantes se basa, en la mayoría de los casos, en la memorización y no se ofrecen oportunidades de mejorar la nota con base en la retroalimentación de los profesores.

Los docentes realizan sobre todo una evaluación sumativa, mediante exámenes y algunas veces mediante proyectos. Podría ser un reflejo de que los profesores tienden a enseñar para el examen, supeditándose a lo que exige el MEP.

Los docentes, en su mayoría, no están aplicando la evaluación formativa, que consiste en evaluar el progreso y los conocimientos del alumno de forma frecuente (tomando en cuenta cada actividad que se propone a los estudiantes) e interactiva (posibilitando su mejora con base en la retroalimentación del profesor). Los estudios muestran que la evaluación formativa es una de las estrategias más efectivas para aumentar el rendimiento escolar y para que los alumnos "aprendan a aprender" (Aguaded, López y Jaén, 2013).

De las afirmaciones anteriores, se deriva la necesidad de ofrecer capacitación a los docentes para que comprendan la importancia de la evaluación formativa y, por ende, el planteamiento de actividades frecuentes, tanto individuales como colaborativas. En ese sentido, la utilización de las TIC ayuda para facilitar este proceso y es fundamental que los profesores aprendan cómo integrarlas.

\section{Uso de las TIC en el aula}

Fue necesario revisar cómo utilizaban las TIC los sujetos participantes del proceso de enseñanza-aprendizaje. Un porcentaje importante (76\%) de estudiantes señaló que el uso de tecnologías como computadora, videos, Google Drive, audios, presentaciones, entre otros, son recursos que podrían influir positivamente en su aprendizaje. Lo anterior, de acuerdo con los sujetos estudiados, les permite participar de estrategias didácticas diversas y acceder a diferentes medios en los que se presenta la información.

Sin embargo, los cuestionarios aplicados reflejaron que el uso de las TIC por parte de los profesores es relativamente escaso. De acuerdo con los resultados arrojados tanto por los profesores como por los estudiantes, el proyector con videos o presentaciones son los recursos que casi siempre se utilizan durante las lecciones. Mientras tanto, el uso de internet, búsquedas en la web y la utilización de herramientas tecnológicas para la comunicación, la creación y la publicación de proyectos es relativamente poca. Igualmente, de acuerdo con los resultados del cuestionario a docentes, la clase magistral es bastante común.

Solamente seis docentes manifestaron que utilizan tecnologías con sus grupos de estudiantes, como correo electrónico o un grupo de Facebook, pero con fines meramente informativos: se cuelgan documentos o avisos. La mayoría de los docentes entrevistados afirma que el obstáculo más importante a la hora de incorporar las TIC en el proceso de enseñanza-aprendizaje es la falta de capacitación sobre el tema. Otro porcentaje (32\%) menciona el gran tamaño de los grupos, el tiempo escaso y la falta de infraestructura tecnológica. Además, un docente mencionó que la aplicación de las tecnologías no asegura un buen rendimiento académico. No obstante, de ser usadas de forma adecuada, cuentan con el potencial para convertirse en aliados con el fin de lograr una mejor calidad del aprendizaje de los estudiantes. Evidentemente, es necesaria una capacitación a los docentes para que ellos puedan integrar pedagógicamente las TIC en su propuesta de enseñanza. 


\section{Formación y actualización docente}

La UNESCO (2008) expresa lo siguiente:

Lograr la integración de las TIC en el aula dependerá de la capacidad de los maestros para estructurar el ambiente de aprendizaje de forma no tradicional, fusionar las TIC con nuevas pedagogías y fomentar clases dinámicas en el plano social, estimulando la interacción cooperativa, el aprendizaje colaborativo y el trabajo en grupo. Esto exige adquirir un conjunto diferente de competencias para manejar la clase. (p.7)

En esta línea es importante preguntarse ¿cómo diseñar y producir actividades con medios didácticos para determinados contenidos y tareas de aprendizaje? En este sentido, es necesario que el docente cuente con conocimientos pedagógicos que le permita crear mejores entornos para el aprendizaje. También, debe aprovechar las tecnologías de manera que se potencien los aspectos de contenidos y los pedagógicos (Churches, 2010).

Ante este panorama, ¿están los docentes del Colegio Calasanz capacitados para desempeñar esas nuevas funciones? $68 \%$ de los docentes ha recibido capacitación sobre estrategias de enseñanza en la asignatura que está a su cargo y tienen interés en buscar alternativas que permitan plantear estrategias didácticas innovadoras. También, $64 \%$ cuenta con alguna formación sobre las TIC como recursos didácticos, pero no están actualizados con los nuevos recursos disponibles para la enseñanza de la asignatura a su cargo. El resto de los docentes (casi 40\%) informa que no ha recibido formación en el tema.

Además, $86 \%$ consideró que la actualización sobre estrategias innovadoras en la didáctica de su asignatura mejoraría los aprendizajes de los estudiantes, y 100\% desea capacitarse y actualizarse sobre estrategias didácticas innovadoras mediante recursos tecnológicos. Lo anterior se observa en la figura 3.

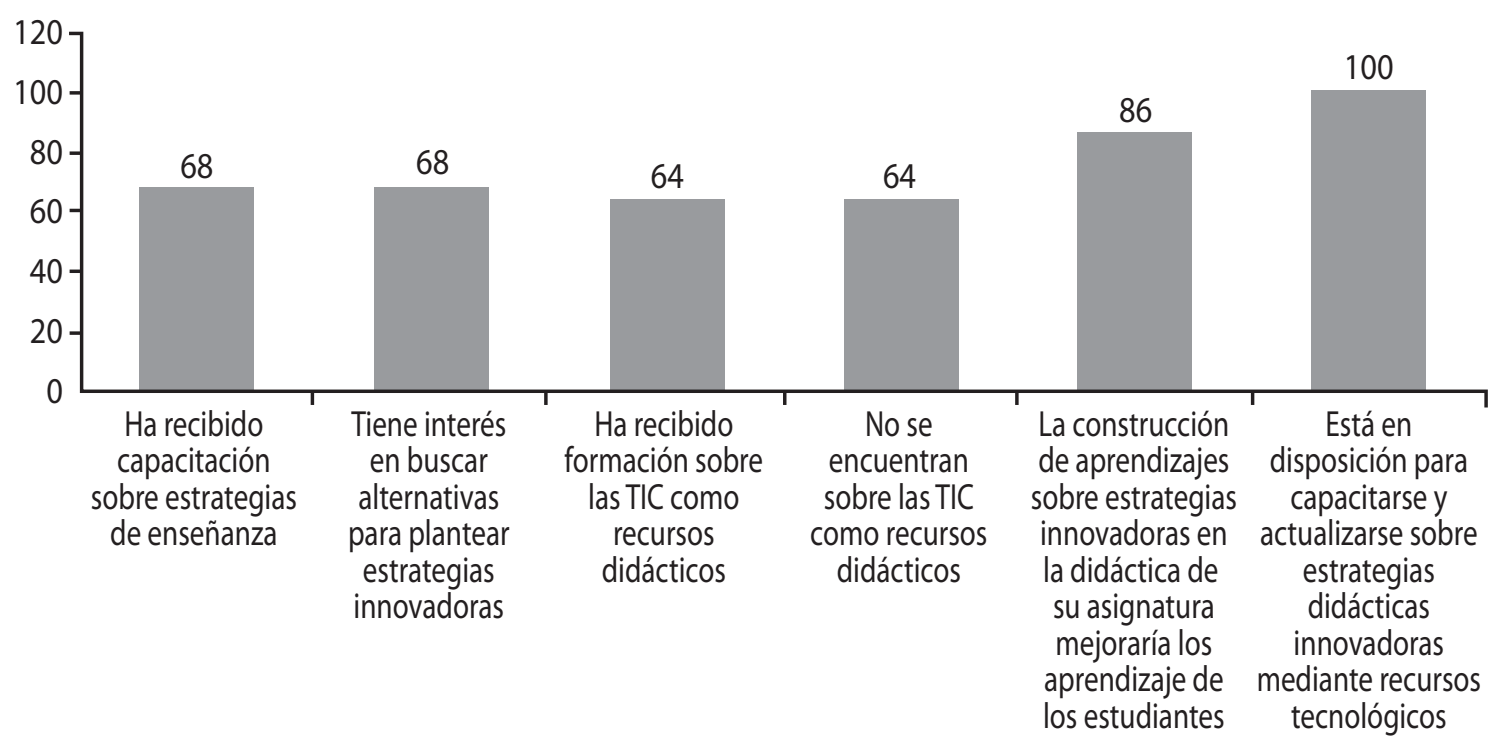

Figura 3. Condiciones de los docentes respecto a las condiciones didácticas de los docentes. Fuente: Anchetta (2015). 
Lo expuesto demuestra que existe un ambiente propicio para iniciar la capacitación de los profesores sobre el tema de la integración de las TIC en el proceso educativo (Tello y Aguaded, 2009; Sáenz, 2011).

\section{CONCLUSIONES}

La investigación realizada permitió concluir que existen diferencias entre lo que los estudiantes necesitan y lo que los docentes ofrecen, a pesar de su disposición e interés. Esto demuestra también que una propuesta con TIC va mucho más allá de la simple inclusión de medios digitales y que sin una investigación exhaustiva de la situación, no habrá resultados exitosos.

Se observó que la mayoría de los estudiantes tienen acceso a internet tanto en su hogar como en su dispositivo móvil, además tienden a pasar mucho tiempo conectados haciendo uso de la web y de sus diversas aplicaciones. Los estudiantes, en orden descendente, prefieren hacer uso de las redes sociales, la búsqueda de información como pasatiempo, la mensajería instantánea, los videojuegos, el correo electrónico y, por último, la búsqueda de información para tareas o trabajos. Estas prácticas de los estudiantes cambian la forma en que se comunican, dialogan, se proyectan en la red como individuos y la manera en que buscan, acceden y discriminan la información.

Ligado a lo anterior, la mayoría de los estudiantes expresaron que el uso de las tecnologías que frecuentan sería de aprovechamiento para mejorar sus aprendizajes, pues implicarían el incorporar estrategias variadas. No obstante, los profesores utilizan más que nada el proyector y las presentaciones en sus lecciones y el porcentaje que usa otras tecnologías es poco. Entre las razones de lo anterior, los docentes expresaron que, pese a que opinan que la inclusión de las TIC podría garantizar mejoras académicas, no disponen de tiempo para hacerlo por el tamaño de los grupos y por la necesidad de cumplir con los objetivos del MEP.

Otro aporte interesante de la investigación fue que no todos los profesores toman en cuenta la motivación como elemento para planificar sus lecciones; además, en ocasiones no evidencian una actitud que invite al interés por parte de los estudiantes, quienes expresaron que solo en algunas ocasiones se sienten motivados para aprender y que, por lo contrario, se aburren en clases y no se interesan en los estudios. Por otro lado, los estudiantes expresaron la necesidad de que los contenidos sean actualizados y relevantes para su vida.

Un porcentaje importante de estudiantes en frecuentes ocasiones olvida lo que fue evaluado en el colegio y siente pocos deseos de continuar estudiando fuera del salón de clase. En este punto corresponde una reflexión de cómo el docente puede desarrollar la habilidad de aprender a aprender, de relacionar al estudiante con su entorno mediante los contenidos expuestos y de resolver problemáticas pertinentes.

De acuerdo con lo propuesto en este trabajo de investigación, las TIC serían un método para que los estudiantes logren lo anterior, puesto que permiten que el aprendizaje se lleve a cabo fuera del salón de clase, son atractivas, motivadoras y se relacionan con las prácticas del estudiantado. Sin embargo, para poder aplicar las tecnologías como recurso didáctico es fundamental una metodología pedagógica pertinente.

De acuerdo con los estudiantes, solamente a veces y algunos de los docentes presentan los contenidos a través de medios como audios, videos e imágenes y los relacionan con el contexto de los alumnos. Por otro lado, los docentes expresaron que sí lo realizan como prácticas frecuentes. Entonces, es importante preguntarse por qué esta no es una práctica presente en la mayoría de los casos y que sea percibida así por los estudiantes. ¿Cuáles son los obstáculos que hallan los docentes para su aplicación?, ¿serán las tecnologías los medios didácticos que ayuden a vencerlos? 
Sobre las actividades de aprendizaje, los estudiantes expresaron que no cuentan con suficiente espacio para criticar, cuestionar, reflexionar y debatir sobre los contenidos. Lo anterior se relaciona con el hecho de que no todos los docentes planifican sus lecciones para que el aprendizaje sea significativo, atractivo y motivador. En su mayoría, la evaluación de los aprendizajes es sumativa e implica la memorización y la repetición de los contenidos y no se brinda la oportunidad de mejorar la calificación. Por ello, se vuelve fundamental que los docentes comprendan la utilidad de la evaluación formativa y cómo pueden aplicarla.

En suma, es importante que la dirección y la administración de la institución educativa se comprometan para que una propuesta integral del proceso de enseñanza-aprendizaje con TIC sea exitosa; es decir, brindándole a los docentes una metodología innovadora, un proceso de capacitación continua y los equipos y los programas informáticos para desarrollar la propuesta, la cual debe fundamentarse en principios pedagógicos pertinentes que tomen en cuenta las características de los estudiantes, los docentes y el contexto.

\section{REFERENCIAS}

Aguaded, J., López, E. y Jaén, A. (2013). Portafolios electrónicos universitarios para una nueva metodología de enseñanza superior. Desarrollo de un material educativo multimedia (MUM). Revista universidad y sociedad del conocimiento. 10(1). Recuperado de http://www.redalyc.org/articulo. oa?id=78025711002

Alemán, L., Sancho, T. y Gómez, M. (2015). Indicadores de calidad pedagógica para el diseño de un curso en línea masivo y abierto de actualización docente. Revista universidad y sociedad del conocimiento. 12(1). Recuperado de http://journals.uoc.edu/index.php/rusc/article/view/ v12n1-aleman-sancho-gomez/v12n1-aleman-sancho-gomez-es

Alemán, L., Sancho, T. y Gómez, M. (2015). Indicadores de calidad pedagógica para el diseño de un curso en línea masivo y abierto de actualización docente. Revista universidad y sociedad del conocimiento. 12(1). Recuperado de http://journals.uoc.edu/index.php/rusc/article/view/ v12n1-aleman-sancho-gomez/v12n1-aleman-sancho-gomez-es

Anchetta, G. (2015). Integración educativa de las TIC en las aulas de secundaria del Colegio Calasanz. Criterios metodológicos y producción de un curso híbrido para la actualización docente. (Tesis de maestría). Universidad Estatal a Distancia, Costa Rica.

Area, M. (2010). El proceso de integración y uso pedagógico de las TIC en los centros educativos. Un estudio de casos. Revista de Educación de la Secretaria de Estado de Educación, Formación profesional y universidades. (352). Recuperado de http://www.revistaeducacion.educacion.es/re352/ re352_04.pdf

Ávalos, B. (2011). Teacher professional development in teaching and teacher education over ten years. Atlanta: Elsevier.

Ayllón, D. (2010). El uso didáctico de internet en secundaria. Las Webquest y las páginas Web. Revista digital de educación y formación del profesorado. 1697-9745. Revista digital de educación y formación del profesorado. España. Centro del Profesorado de Córdoba. Recuperado de http://revistaeco. cepcordoba.org/index.php?option=com_content\&view=article\&id=31:el-uso-didactico-de-internet-en-secundaria-las-Webquest-y-la-pagina-Web\&catid=1:articulos\&Itemid=5

Boekarts, M. (2006). Motivar para aprender. Ginebra: Universidad pedagógica nacional.

Bono, E. (2011). La calidad de los aprendizajes en la enseñanza secundaria. Revista de currículum y formación del profesorado. ISSN 1989-639X. Recuperado de http://www.ugr.es/ recfpro/rev152COL10.pdf 
Bravslasky, C. (2004). Diez factores para una educación de calidad para todos en el siglo XXI. Madrid: Fundación Santillana.

Clavijo, G. (2008). La evaluación del proceso de formación. Colombia aprende. Recuperado de http:// www.colombiaaprende.edu.co/html/productos/1685/articles-178627_ponen7.pdf

Churches, A. (2010). Educational origami. Recuperado de http://edorigami.wikispaces.com/ The+Digital+Citizen

Dobles, M., Zúñiga, M., García, J. (2013). Investigación en educación. San José: EUNED.

Fournier, M. y Grillo, M. (2010). Conocimientos, actitudes y prácticas asociados al uso de Internet en los adolescentes: informe sobre estudio CAP en colegios de la región metropolitana. San José: Fundación Paniamor.

González, J. (2000). El paradigma interpretativo en la investigación social y educativa: nuevas respuestas para viejos interrogantes. Universidad de Sevilla. Recuperado de http://institucional.us.es/revistas/ cuestiones/15/art_16.pdf

Hernández, R., Fernández, C. y Baptista, P. (2010). Metodología de la investigación. México: McGraw Hill.

Henríquez, P. y Organista, J. (2009). Definición y estimación de tipos y niveles de uso tecnológico: una aproximación a partir de estudiantes de recién ingreso a la universidad. Revista electrónica de tecnología educativa. (30)1. Recuperado de http://edutec.rediris.es/Revelec2/revelec30/articulos_n30_ pdf/Edutec-e30_Henriquez_Organista.pdf

Maglione, C. y Varlotta, N. (2010). Investigación, gestión y búsqueda de información en Internet. Buenos Aires: Conectar Igualdad.

Maguire, L. (2008). Developing distance education policy within a state system of higher education: the faculty perspective. (Tesis doctoral). The Pennsylvania State University. Recuperado de http://search. proquest.com.talamanca.uned.ac.cr/docview/304496317/fulltextPDF/140CE00E78504A21PQ/3?ac countid $=37042$

Mella, E. (2003). La educación en la sociedad del conocimiento y del riesgo. Revista enfoques educacionales. 5(1). Recuperado de http://www.facso.uchile.cl/publicaciones/enfoques/07/Mella LaEducacionenlaSociedaddelConocyelCambio.pdf

Meza, J. (2013). Metodología para proyectos de formación virtual. Libro electrónico, Organismo de Cooperación Alemana (GIZ). Recuperado de https://gc21.giz.de/ibt/var/app/wp342P/1522/index. php/organisationen/best-practice/?lang=de

Organización para la cooperación y desarrollo económicos. (2010). Panorama de la educación 2010. Madrid: Fundación Santillana.

Pegalajar, M. (2015). Metodología docente en el máster de formación del profesorado en educación secundaria:valoración del alumnado. Revista universidad y sociedad del conocimiento.12 (3). Recuperado http://journals.uoc.edu/index.php/rusc/article/view/v12n3-pegalajar/v12n3-pegalajar-es

Penzo, W., Fernández, V., García, I., Gros, B., Pagés, T., Roca, M., Vallés, A. y Vendrell, P. (2010). Guía para la elaboración de las actividades de aprendizaje. Barcelona: Editorial OCTAEDRO.

Pérez, R., Blanca, E., Brenes, C., Quesada, S., Rumoroso, A. y Torres, D. (2008). Uso de las tecnologías de la comunicación e información en jóvenes de 12 a 18 años del Gran Área Metropolitana. Instituto de investigaciones psicológicas, Universidad de Costa Rica. Recuperado de http://www.programatecnologiasi.org/natic/wp-content/uploads/2013/PANIAMOR_2008.pdf

Prensky, M. (2010). Nativos e inmigrantes digitales. Institución educativa SEK. Recuperado de http://www. marcprensky.com/writing/Prensky-NATIVOS\%20E\%20ININMIGRANTES\%20DIGITALES\%20(SEK).pdf

Prensky, M. (2010). Nativos e inmigrantes digitales. Institución educativa SEK. Recuperado de http://www. marcprensky.com/writing/Prensky-NATIVOS\%20E\%20ININMIGRANTES\%20DIGITALES\%20(SEK).pdf 
Programa Estado de la Nación (2015). Informe estado de la educación. Recuperado de http://www.estadonacion.or.cr/educacion2015/assets/cap-3-ee-2015.pdf

PROSIC (2009). Brecha digital en la educación secundaria: el caso de los estudiantes costarricenses. Programa sociedad de la información y comunicación. Recuperado de http://www.prosic.ucr.ac.cr/sites/default/files/documentos/estudiantes.pdf

Raventós, P. (2007). Uso educativo de las TIC en Secundaria: algunos ejemplos prácticos. Grupo de tecnología educativa. Recuperado de http://tecnologiaedu.us.es/cuestionario/bibliovir/utic.pdf

Rioseco, M. y Romero, R. (s.f.). La contextualización de la enseñanza como elemento facilitador del aprendizaje significativo. Universidad de Concepción. Recuperado de http://www.oei.es/equidad/ rioseco3.PDF

Sáenz, J. (2011). Opiniones y práctica de los docentes con respecto al uso pedagógico de las Tecnologías de la Información y Comunicación. Revista electrónica de investigación y docencia. 1988-2446. Recuperado de http://www.ujaen.es/revista/reid/revista/n5/REID5art5.pdf

Sánchez, H. (19 de julio de 2014). Comunicación personal.

Sánchez, J. (s.f.). Integración curricular de las TIC: conceptos e ideas. Universidad de Chile. Recuperado de http://www.c5.cl/mici/pag/papers/inegr_curr.pdf

Tedesco, J. (2011). Los desafíos de la educación básica en el siglo XXI. Revista iberoamericana de educación. 55. P.31-47. Recuperado de http://www.rieoei.org/rie55a01.pdf

Tello, J. y Aguaded, J. (2009). Desarrollo profesional docente ante los nuevos retos de las tecnologías de la información y la comunicación en los centros educativos Revista de Medios y Educación. № 34 Enero 2009. Universidad de Huelva. España. Recuperado de http://www.academia. edu/3573859/DESARROLLO_PROFESIONAL_DOCENTE_ANTE_LOS_NUEVOS_RETOS_DE_LAS_ TECNOLOG\%C3\%8DAS_DE_LA_INFORMACI\%C3\%93N_Y_LA_COMUNICACI\%C3\%93N_EN_LOS_ CENTROS_EDUCATIVOS

UNESCO (1996). La educación encierra un tesoro. Informe a la UNESCO de la Comisión Internacional sobre la Educación para el Siglo XXI. Editorial Santillana. Recuperado de http://unesdoc.unesco.org/ images/0010/001095/109590so.pdf

UNESCO (2008). Estándares de competencia en TIC para docentes. Londres. Recuperado de http://www. oei.es/tic/UNESCOEstandaresDocentes.pdf

UNESCO (2013). Uso de las TIC en educación en América Latina y el Caribe: análisis regional de la integración de las TIC en la educación y de la aptitud digital (e-readiness). Recuperado de http://www.uis.unesco. org/Communication/Documents/ict-regional-survey-lac-2012-sp.pdf

UNICEF (2010). Educación secundaria: derecho, inclusión y desarrollo. Desafíos para la educación de los adolescentes. Recuperado de http://www.unicef.org/argentina/spanish/Educacion_Secundaria(1).pdf 